\title{
Fingerprinting Lymph Node Metastases
}

\author{
W. Fraser Symmans, $\mathrm{MD}^{1}$ and Gabriel N. Hortobagyi, $\mathrm{MD}^{2}$
}

\begin{abstract}
${ }^{1}$ Department of Pathology, The University of Texas M. D. Anderson Cancer Center, Houston, TX 77030-1439, USA
${ }^{2}$ Department of Breast Medical Oncology, The University of Texas M. D. Anderson Cancer Center, 1515 Holcombe Blvd., Box 301439, Unit 1354, Houston, TX 77030-1439, USA
\end{abstract}

Within a decade of its invention, the technology commonly termed "DNA fingerprinting" transformed forensic science and had a huge impact on the legal system. Thanks to this procedure, which combines polymerase chain reaction for sequence amplification and capillary electrophoresis as a sensitive high-throughput detection method, it is now practical to evaluate small amounts of degenerated DNA and interrogate multiple known sites of highly variable genetic sequence, known as microsatellites. In forensic sciences, a perfect match of sequence at multiple microsatellites is used to assign identity with near certainty. This technology is applicable to the degraded DNA of formalin-fixed tissues and has been successfully applied to breast cancers by Ellsworth et al. ${ }^{1}$ In the article by Becker et al., ${ }^{2}$ this same research group has interrogated 26 microsatellite loci scattered across the genome and has applied statistical models to estimate the similarity between the primary tumor and each nodal metastasis in 26 patients with node-positive breast cancer. This work builds on previous studies that have applied techniques such as DNA ploidy, immunohistochemical markers, fluorescent in situ hybridization, comparative genomic hybridization, and gene expression profiling to explore the clonal progression model of cancer due to its characteristic genomic instability.

The investigators attempted to map the order of each metastasis. Assuming that allelic imbalances (AIs) of microsatellite genetic sequence continue to

Published online March 14, 2008.

Address correspondence and reprint requests to: Gabriel N. Hortobagyi, MD; E-mail: ghortoba@mdanderson.org

Published by Springer Science+Business Media, LLC @ 2008 The Society of Surgical Oncology, Inc. accrue during tumor progression (but do not disappear), they supposed that the most recent nodal metastases would have the most AIs and the highest similarity to the primary tumor sample, whereas older metastases would have fewer AIs. This is the basis of the heritage maps that they present as possible timelines of nodal metastases in each patient. Of course, there is no way of proving the timing of each metastasis in a patient at the time of surgery, but the concept is intriguing.

A number of unaccounted factors could also affect this model. First, primary breast cancers are often biologically heterogeneous, such that the single sample of each primary tumor evaluated in this study might not fully represent that heterogeneity. The frequent observation in some nodal metastases of AI at genetic sites that were normal in the primary tumor sample could be explained by intratumoral heterogeneity that was not appreciated from one sample. Second, it is likely that metastases also exhibit genetic instability during their growth. This could produce new genetic sites of AI in some metastases, but not in the primary tumor. Third, because the lymphatic drainage routes persist, it is likely that nodal metastases continue to be seeded by the primary tumor (and even upstream nodal metastases) such that new clones are periodically added to an existing metastatic population. Combinations of these possible factors would add to the chaos.

In future studies, it would be interesting to apply this technology to current specimens from sentinel lymph nodes, from which certain inferences could be made about what constitutes a recent nodal metastasis, or about possible patterns of involvement. One could also explore whether metastases have greater genetic instability with more advanced nodal stage, possibly adding to the prognostic relevance. 
Questions that arise from this article also relate to clinical management. To what extent is genomic instability a reflection of tumor phenotype, and to what extent is the rate of genomic instability a factor in prognosis and treatment resistance? The first question cannot be addressed by using only 26 selected cases, but it is potentially answerable. The authors suggest that genomic instability may relate to treatment failure, but proof is not forthcoming. Indeed, we can ponder that the incurability of stage IV disease could be attributable to accelerated genetic chaos in addition to the rate of growth and overall burden of disease. This technology can be applied to archival tissue samples, and probably also cytologic samples with alcohol-based fixation, and so could be used to determine the extent and rate of genomic instability during the course of disease progression in patients who have undergone biopsy at times of relapse. Temporal heterogeneity is certain to be a relevant biological factor that can only be evaluated in serial samples. Indeed, the biology of distant metastasis is a high priority for translational breast cancer research.

When one considers that 26 microsatellites represent a few tiny specks on the canvas of the cancer genome, it becomes apparent that we know little of a complex and evolving picture. However, studies such as this will shape new hypotheses and help to direct future research.

\section{REFERENCES}

1. Ellsworth RE, Ellsworth DL, Lubert SM, et al. High-throughput loss of heterozygosity mapping in 26 commonly deleted regions in breast cancer. Cancer Epidemiol Biomarkers Prev 2003; 12:915-9.

2. Becker TE, Ellsworth RE, Deyerman B, et al. The genomic heritage of lymph node metastases: implications for clinical management of patients with breast cancer. Ann Surg Oncol 2008. 